07

\title{
Применение числовых характеристик рекуррентных графиков для анализа механической поврежденности материалов
}

\author{
(C) В.Л. Гиляров \\ Физико-технический институт им. А.Ф. Иофрфе РАН, \\ Санкт-Петербург, Россия \\ E-mail: Vladimir.Hilarov@mail.ioffe.ru
}

(Поступила в Редакцию 16 февраля 2017 г.)

Исследован отклик материала со случайным равномерным распределением пор на звуковой импульс. Изучено поведение числовых характеристик рекуррентных графиков (RQA) нормальной компоненты вектора смещения в зависимости от степени поврежденности. Показано, что RQA-параметры могут быть весьма информативными для звуковой дефектоскопии.

Работа выполнена при финансовой поддержке РФФИ (проект № 16-05-00237 А).

DOI: 10.21883/FTT.2017.09.44848.044

Предупреждение природных и техногенных катастроф представляет собой чрезвычайно важную задачу. В частности, актуальным является проведение мониторинга работы агрегатов и конструкций с целью выявления неполадок в их функционировании. К таким методам мониторинга относится ультразвуковая дефектоскопия, представляющая собой один из самых распространенных способов неразрушающего контроля. В то же время разрушение материалов представляет собой нелинейный и нестационарный процесс, и обычные методы, используемые в дефектоскопии, могут оказаться неэффективными. Например, частоты, амплитуды и формы собственных колебаний могут изменяться в процессе измерения за счет нестационарности или быть неопределимыми в силу отсутствия принципа суперпозиции в нелинейных системах и, как следствие, неприменимости преобразования Фурье к таким системам вообще. Этих недостатков лишен используемый в настоящей работе метод рекуррентных графиков, специально разработанный для изучения поведения во времени нелинейных динамических систем.

В работе [1] было предложено использовать метод рекуррентных графиков (RP) [2], в частности следующий из этого метода количественный рекуррентный анализ (RQA) [3], для изучения поврежденности материалов. В качестве модели материала с дефектами в [1] выступала стальная плита с боковыми надрезами разной длины. В настоящей работе в качестве такого материала рассматривается плита со случайным распределением пор.

Рекуррентный график определяется как множество точек, подчиняющихся соотношению

$$
R_{i, j}(r, m)=\theta\left(\varepsilon_{i}-\left\|\mathbf{X}_{i}(m)-\mathbf{X}_{j}(m)\right\|\right),
$$

где $\mathbf{X}_{i}(m)$ - фазовая траектория динамической системы в $m$-мерном фазовом пространстве, $\theta-$ функция Хевисайда, а \|\| - норма вектора. Таким образом, рекуррентный график представляет собой двумерное множество точек, отвечающих соотношению (1), состоящее из нулей и единиц, причем близкие (рекуррентные) точки принято отображать черным цветом, а далекие белым. Близость точек определяется параметром $\varepsilon_{i}$.

Можно обобщить определение рекуррентного графика [4] на случай совместного изменения двух динамических систем

$$
C R_{i, j}(r, m)=\theta\left(\varepsilon_{i}-\left\|\mathbf{X}_{i}(m)-\mathbf{Y}_{j}(m)\right\|\right) .
$$

Полученный таким способом кросс-рекуррентный график (CRP) позволяет изучить корреляции в поведении двух различных систем.

В настоящей работе метод рекуррентных графиков применяется для изучения реакции материала на импульс механической силы. Объект исследования представляет собой прямоугольную стальную пластину размером $2.4 \times 1.6 \mathrm{~m}$ с толщиной $0.01 \mathrm{~m}$. Рассматривается плоская нестационарная задача теории упругости. На двух длинных торцах установлены точечные звуковые датчики, один из которых считается излучающим (с координатами $(0,-0.8))$, а три других (на противоположном торце) - приемными (рис. 1, $a$ ). Координаты приемных датчиков следующие: $(-1,0.8),(0,0.8),(0.6,0.8)$. На излучающий датчик подается прямоугольный импульс силы шириной $1 \mathrm{~ms}$, сглаженный за время $10^{-5} \mathrm{~s}$, в направлении вертикальной оси $y$. На приемных датчиках записывается временна́я зависимость нормального смещения $u_{y}$ во временно́м интервале $0-0.01 \mathrm{~s}$, порожденного этим импульсом. Боковые концы пластины фиксировались (вектор смещения задавался равным нулю). Предполагалось, что деформации настолько малы, что материал остается линейным.

Внутри пластины делались круглые вырезы (поры). Координаты пор задавались генератором псевдослучайных чисел с равномерным распределением, а их радиусы также предполагались равномерно распределенными в диапазоне 0-0.05 m. Расчет производился при разных 

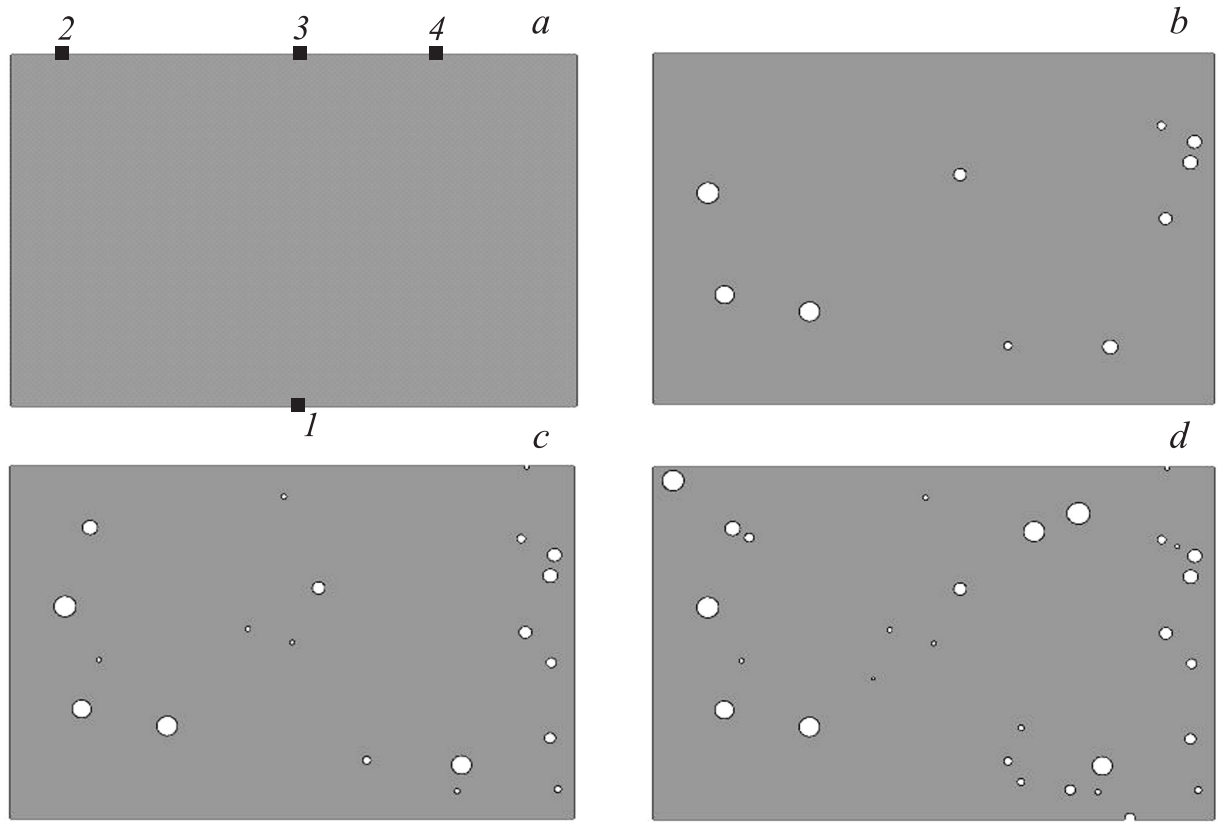

$e$
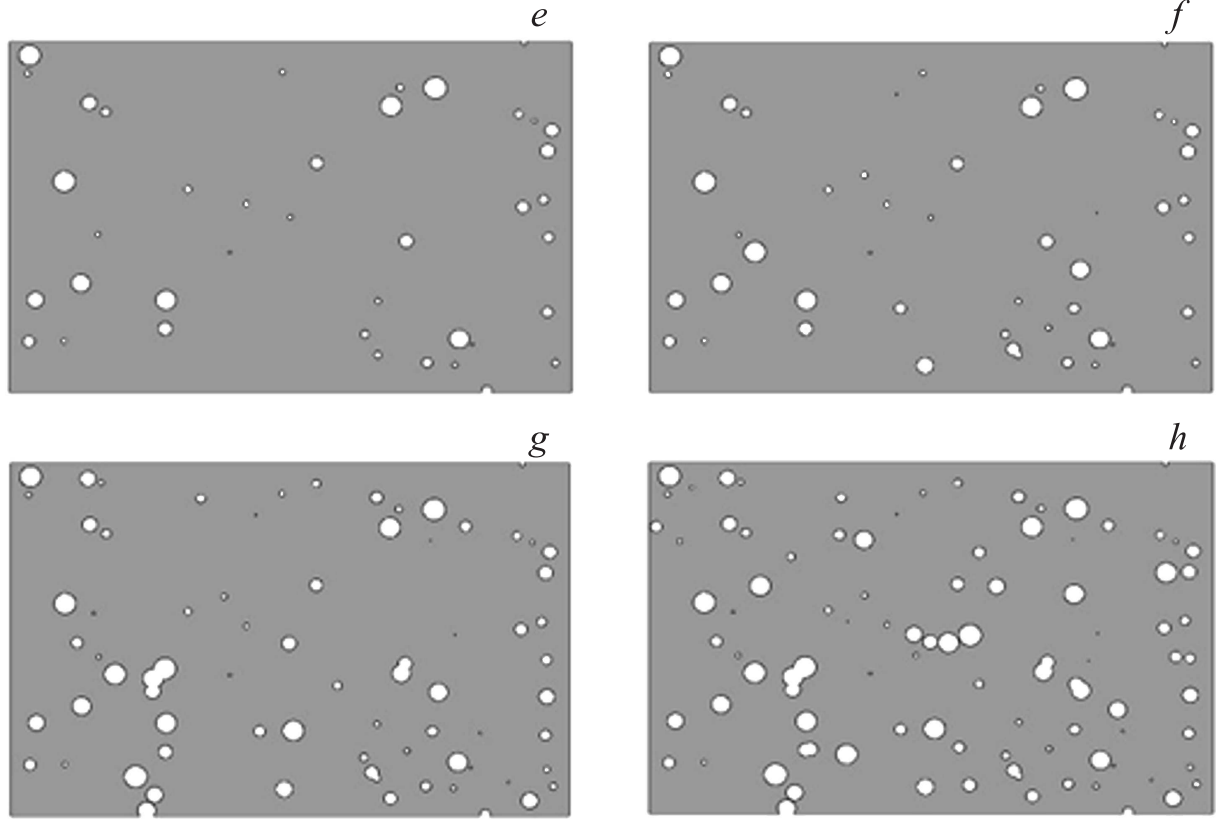

Рис. 1. $a-$ бездефектный образец и места расположения датчиков излучающего $(1)$ и приемных $(2-4) ; b-h-$ накопление дефектов в пластине.

степенях дефектности пластины. Дефектность (поврежденность) определялась по формуле

$$
D=\left(1-\frac{S_{d}}{S_{0}}\right) \cdot 100 \%
$$

Здесь $S_{0}-$ площадь бездефектной пластины, $S_{d}-$ площадь пластины с дефектами. Вид пластины с различным числом дефектов приведен на рис. $1, b-h$. Расчет проводился методом конечных элементов. Шаг интегрирования по времени составлял $10^{-5} \mathrm{~s}$. При таком выборе временно́го шага и соответствующем пространственном разбиении полученные решения обладали хорошей схо- димостью и были устойчивы во всем диапазоне интегрирования даже без учета затухания.

На основе полученных временны́х зависимостей нормальной компоненты вектора смещения для разных уровней поврежденности строились рекуррентные графики вида (1) и (2), где в качестве векторов $\mathbf{X}$ и $\mathbf{Y}$ выступали $y$-компоненты смещений на датчиках 2-4. В качестве примера на рис. 2 представлены CRP величины $u_{y}$ на датчике 3 для исходного (неповрежденного) и двух поврежденных образцов $(D \approx 2$ и $\approx 11 \%)$ при размерности $m=1$ и пороге рекуррентности $\varepsilon_{i}=\varepsilon=0.1 \forall i$. Начальный участок RP отличается от остального RP, 

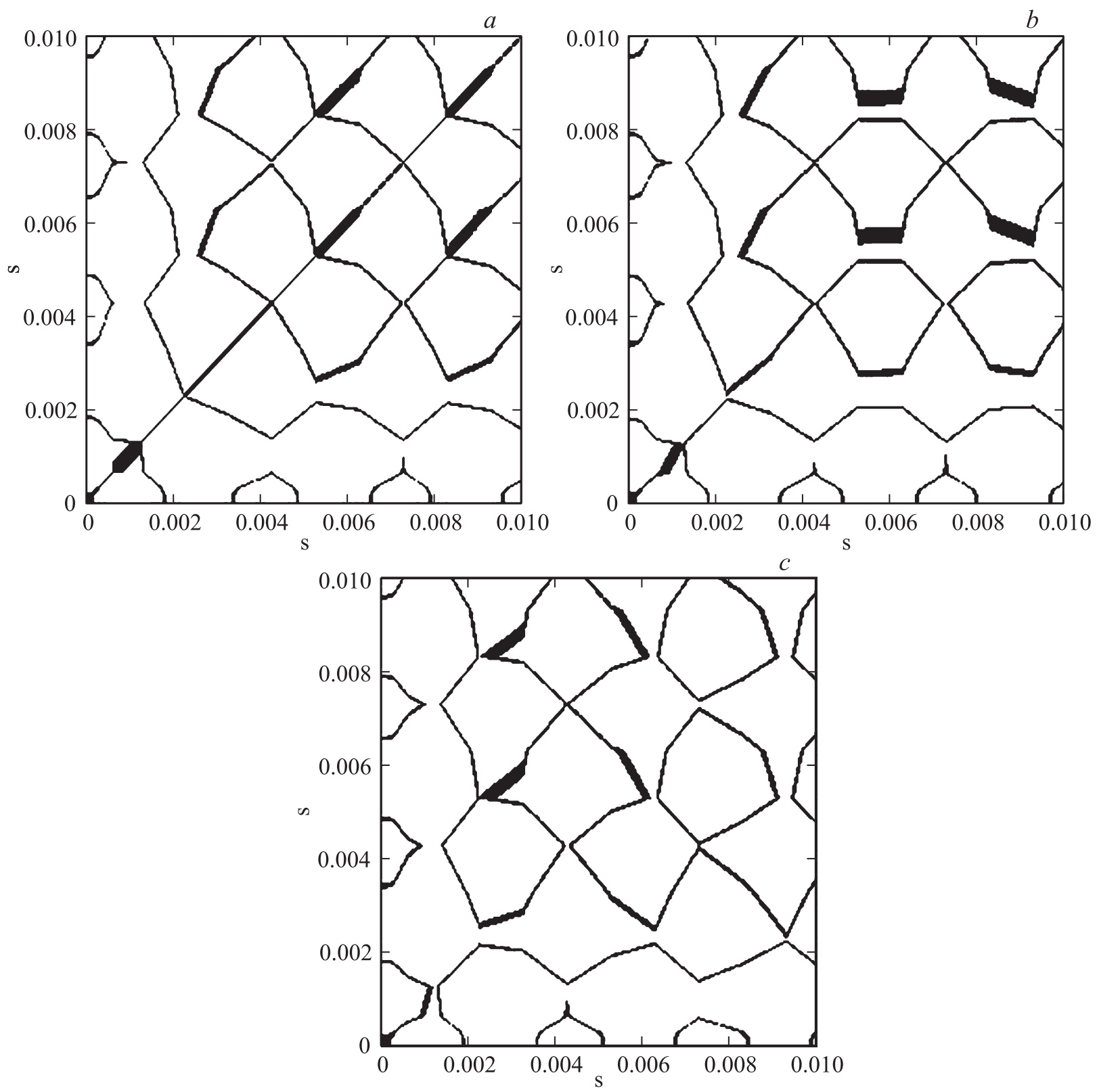

Рис. 2. Рекуррентные графики для бездефектного образца $(a)$ и двух дефектных: $D \approx 2(b)$ и $\approx 11 \%(c)$.

что связано с нестационарностью процесса, так как через $10^{-3} \mathrm{~s}$ после начала отсчета времени действие силового импульса прекращается. Отсутствие хаотически разбросанных по RP отдельных точек указывает на отсутствие стохастичности. Конечный участок имеет черты, характерные для периодических систем с суперпозицией гармонических колебаний [5].

Данные для неповрежденного образца использовались как опорные при построении кросс-рекуррентных графиков на каждом из датчиков при различной степени поврежденности. Для количественного описания изменений RP при увеличении дефектности материала применялся количественный рекуррентный анализ (RQA). Для построения RP и расчета RQA использовался свободно распространяемый пакет программ CRP Toolbox [6]. Beличина порога рекуррентности $\varepsilon$ выбиралась из условия приблизительного равенства плотности рекуррентных точек $1 \%$ и составляла $\varepsilon \approx 0.05-0.1$.

На рис. 3 приведены зависимости некоторых параметров RQA от степени дефектности образца, рассчитанной по формуле (3), для трех приемных датчиков, а также изменение первых десяти собственных частот рассматриваемой пластины. Результаты расчета RQA приведены для размерности пространства $m=1$ для нормальных компонент смещений $u_{y}$ на трех приемных датчиках. Видно, что при случайном равномерном (изотропном) распределении пор различие RQA-параметров на разных приемных датчиках незначительно. Исполь- 

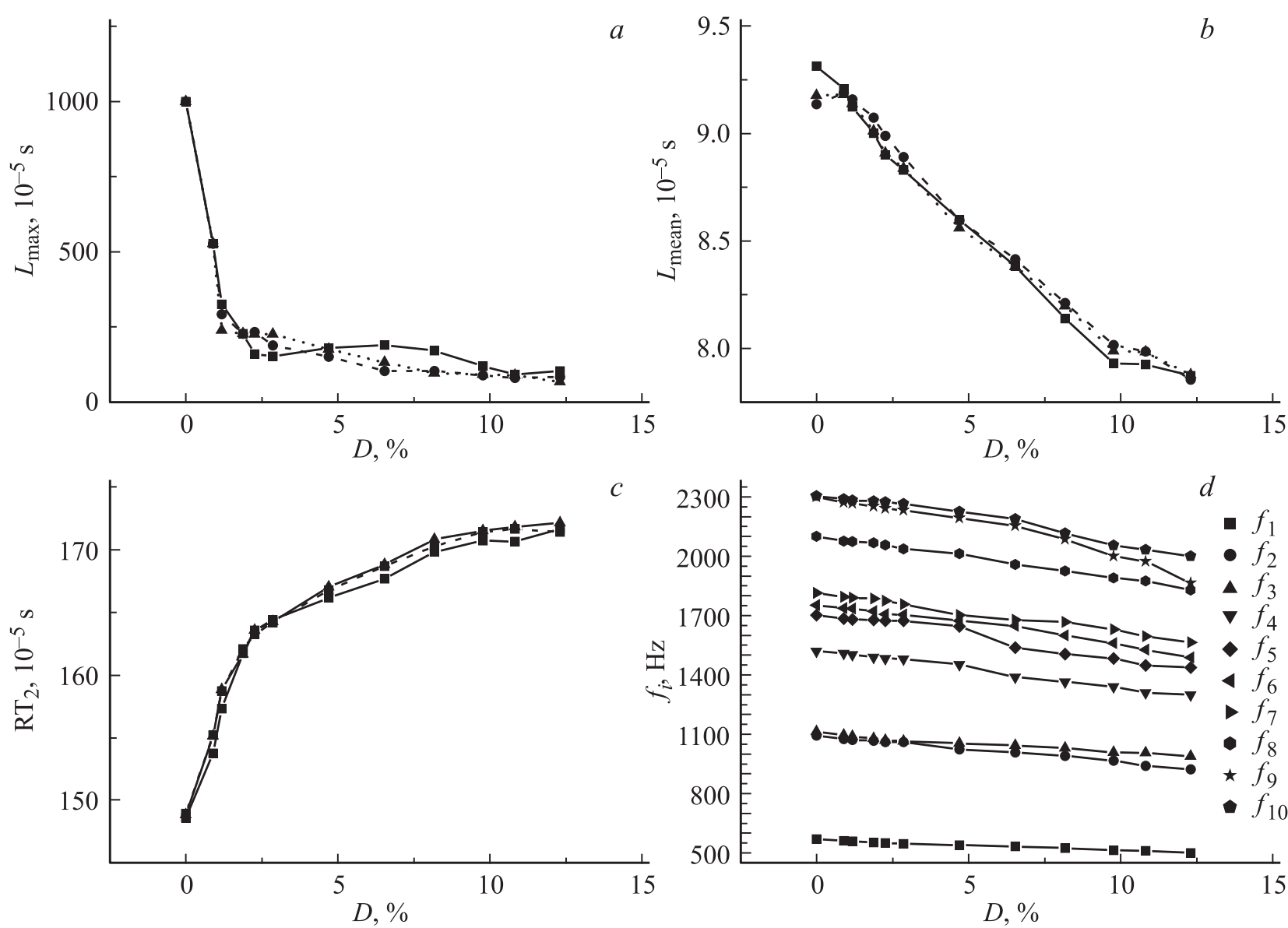

Рис. 3. Зависимости RQA-параметров для трех приемных датчиков: $L_{\max }(a), L_{\operatorname{mean}}(b), \mathrm{RT}_{2}(c)$, а также частот десяти нормальных мод $(d)$ от степени дефектности образца.

зование нескольких датчиков необходимо при изучении анизотропии образования дефектов. Изменение частот не превышает $11-20 \%$, в то время как изменение RQA-параметров может достигать десятков (уровень возврата RR 39\%) и даже сотен процентов (средняя $L_{\text {mean }}$ и максимальная $L_{\max }$ длина диагональных линий, параллельных главной диагонали). Некоторые параметры, такие как RR или энтропия диагональных линий оказались очень чувствительными к величине порога рекуррентности $\varepsilon$. В то же время детерминизм уменьшается при изменении дефектности очень незначительно $(\sim 7 \%)$. Аналогичные расчеты проводились для случая $m=3$, а также при фиксированном числе ближайших соседей (метод FAN [6]). В случае $m=3$ в качестве компонент векторов фазового пространства использовались данные с трех приемных датчиков. Поведение рассматриваемых RQA-параметров при этом качественно не изменяется.

Параметры $L_{\text {mean }}$ и $L_{\max }$ оказываются наиболее устойчивыми при изменении $\varepsilon$, что позволяет сделать вывод о том, что они являются наиболее подходящими для использования в звуковой дефектоскопии при выявлении множественных дефектов на начальных стадиях делокализованного накопления дефектов в процессе разру- шения. Особо следует отметить такую характеристику, как время возврата второго рода $\left(\mathrm{RT}_{2}\right)$, приведенную на рис. 3,c, поскольку она непосредственно отвечает времени возврата Пуанкаре [6]. Увеличение времени возврата при росте дефектности связано с уменьшением собственных частот колебаний и хаотизацией системы. Примечательно, что наибольшие изменения параметров RQA происходят именно в области малой дефектности.

Таким образом, параметры рекуррентных графиков могут служить весьма информативными характеристиками для звуковой и ультразвуковой дефектоскопии.

\section{Список литературы}

[1] J.M. Nichols, S.T. Trickey, M. Seaver. Mechanical Systems Signal Processing 20, 421 (2006)

[2] J.-P. Eckmann, S.O. Kampost, D. Ruelle. Europhys. Lett. 4, 973 (1987).

[3] J.P. Zbilut, C.L. Webber. Phys. Lett. A 171, 199 (1992).

[4] J.P. Zbilut, A. Giuliani, C.L. Webber, J. Phys. Lett. A 246, 122 (1998).

[5] N. Marwan, J. Kurths. Phys. Lett. A 336, 349 (2005).

[6] N. Marwan, M.C. Romano, M. Thiel, J. Kurths. Phys. Rep. 438, 237 (2007). 